\title{
Evolutionary optimization algorithms for nonimaging optical design
}

\section{Ángel García-Botella, Daniel Vázquez-Moliní, Berta Garcia-Fernandez, Antonio Álvarez Fernandez-Balbuena}

Ángel García-Botella, Daniel Vázquez-Moliní, Berta Garcia-Fernandez, Antonio Álvarez Fernandez-Balbuena, "Evolutionary optimization algorithms for nonimaging optical design," Proc. SPIE 11120, Nonimaging Optics: Efficient Design for Illumination and Solar Concentration XVI, 111200M (9 September 2019); doi: 10.1117/12.2529180

EDent: SPIE Optical Engineering + Applications, 2019, San Diego, California, United States 


\title{
Evolutionary optimization algorithms for nonimaging optical design
}

\author{
Angel García-Botella ${ }^{1}$, Daniel Vázquez-Moliní ${ }^{2}$, Berta Garcia-Fernandez ${ }^{12}$, Antonio Álvarez \\ Fernandez-Balbuena ${ }^{1}$, \\ ${ }^{1}$ Departamento de Ingenieria y Gestion Forestal y Ambiental, Universidad Politécnica de Madrid, \\ ETSI de Montes. Ciudad Universitaria s/n 28040 Madrid (SPAIN). e-mail: angel.garciab@upm.es \\ ${ }^{2}$ Departamento de Óptica, Universidad Complutense de Madrid, Fac de Óptica y Optometría. Arcos \\ de Jalón, 118, 28037 Madrid (SPAIN).
}

\begin{abstract}
Evolutionary optimization algorithms have been recently introduced as nonimaging optics design techniques. Unlike optimization of imaging systems, non sequential ray tracing simulations and complex non centred systems design must be considered, adding complexity to the problem. The Merit Function (MF) is a key element in the automatic optimization algorithm, nevertheless the selection of each objective's weight, $\left\{w_{i}\right\}$, inside merit function needs a previous trial and error process for each optimization. The problem then is to determine appropriate weights value for each objective. In this paper we propose a new Dynamic Merit Function, $D M F$, with variable weight factors $\left\{w_{i}(n)\right\}$. The proposed algorithm, automatically adapts weight factors, during the evolution of the optimization process. This dynamic merit function avoids the previous trial and error procedure selecting the right merit function and provides better results than conventional merit functions $(C M F)$. Also we analyse the Multistart optimization algorithm applied in the flowline nonimaging design technique.
\end{abstract}

\section{1.- Introduction}

Evolutionary optimization techniques have been introduced in the design of nonimaging systems recently [1]. Non sequential raytrace tools and complex, non centred, optical systems were the main impediment to extensive application of automatic optimization techniques to nonimaging optics problems. Nowadays, improvements in hardware and software capabilities allows the implementation of optimization utilities in software packages [2, 3] becoming a powerful tool in the nonimaging design problem. The main steps in the nonimaging optimization procedure are: first the parameterization of optical system, including the definition of the constraints in the parameters; second the definition of the merit function $(M F)$ to be minimized or maximized [4]; and third the selection of the optimization algorithm, in particular Nelder-Mead algorithm produces robust and convergent method in nonimaging optimization problem [5].

We focus our attention in the merit function, as it has the role to drive the optimization procedure. Then, improvements in the capabilities of merit function will improve the results of the optimization procedure. The most common way to build merit functions involves the weighted sum of squares of the differences between a set of objectives and their associated target values [6],

$$
M F=\sum_{i} w_{i}\left(V_{i}-T_{i}\right)^{2},
$$

where $w_{i}$ is the weight factor for the $i^{\text {th }}$ objective, $V_{i}$ is the value of the $i^{\text {th }}$ objective and $T_{i}$ is the target value of the $i^{\text {th }}$ objective. Equation (1) shows direct influence of the weight factors in the $M F$

\footnotetext{
Nonimaging Optics: Efficient Design for Illumination and Solar Concentration XVI, edited by Roland Winston, Eli Yablonovitch, Proc. of SPIE Vol. 11120, 111200M · C) 2019 SPIE CCC code: $0277-786 \mathrm{X} / 19 / \$ 21 \cdot$ doi: $10.1117 / 12.2529180$
} 
and therefore in the optimization procedure. Commonly the weights factors $\left\{w_{i}\right\}$ are manually adjusted by trial and error procedure [7], this non-optimal situation suggest the need to study methods for automatic adjustments of the weight factors $\left\{w_{i}\right\}$. In this paper we propose a new type of merit functions, Dynamic Merit Functions (DMF), which automatically adjust the weight factors $\left\{w_{i}\right\}$ during the progress of optimization procedure. The variation of weight factors modifies the optimization problem, and $D M F$ becomes a global optimization method [8].

\section{2.- Dynamic Merit Function (DMF)}

For nonimaging design the most prevalent objectives to be optimized are the efficiency, uniformity, angular emission, concentration factor, etc. [7, 9], and normally all of them must be optimized at the same time. Along this paper the efficiency and uniformity are selected as objectives of the $M F$ as they conform two of the most typical parameters involved in illumination systems. The efficiency measures the flux reaching the detector screen divided by emitted flux. While uniformity is calculated as the mean irradiance value divided by the maximum radiance at the detector screen.

$$
\eta=\frac{\Phi_{\text {detector }}}{\Phi_{\text {emitted }}}, \quad U=\frac{\bar{E}}{E_{\max }}
$$

But the right balance between each one of these objectives in the optimization procedure, and then, the right choose of the Merit Function is still a trial and error process [10], which depends on the particular problem to be considered. To avoid this misfunction we propose a Dynamic Merit Function which automatically modifies the weight of each objective $\left\{w_{i}(n)\right\}$ as the optimization procedure advances.

$$
\left.\left.D M F=2-\left[w_{\eta}(n) \eta+w_{U}(n) U\right)\right]=2-[\lambda(n) \eta+(2-\lambda(n)) U)\right],
$$

where $\eta$ is the efficiency of the system and $U$ is the uniformity, $w_{\eta}$ is the weight factor for efficiency and $w_{U}$ is the weight factor for uniformity, $n$ is the iteration number of the DMF optimization, and $\lambda$ defines the constraint that the weight factors must accomplish:

$$
w_{\eta}(n)+w_{U}(n)=2, w_{\eta}(n)=\lambda(n), w_{U}(n)=2-\lambda(n), \lambda(n) \epsilon[0,2] .
$$

The conventional merit function $(C M F)$ is composed by fixed weight values. An unique $D M F$ iteration comprises several $C M F$ iterations denoted by $m$ index (fig. 1 ). The $C M F$ concludes under two self-contained condition: first condition is met if the $C M F$ iteration $m$ reaches its maximum value $(M)$, and the second condition is specified by means of the tolerance parameters, that establish the minimum variation of the MF considered, TolFun, and the minimum optimization's parameter variation, Tolx. The variations of the $M F$ and the optimization variables have to be simultaneously less than the TolFun and Tolx (respectively) to end the CMF optimization.

The algorithm, aimed to balance the weight of each objective, compares the obtained values of both objectives $(\eta(n), U(n))$ and adds a quantity $D / n$ to the weight factor of the lower objective and subtracts the same value to the upper objective:

$$
\left\{\begin{array}{l}
\text { if }(\eta(n)>U(n)) \Rightarrow w_{\eta}(n+1)=w_{\eta}(n)-\frac{D}{n}, w_{U}(n+1)=w_{U}(n)+\frac{D}{n} \\
\text { if }(U(n)>\eta(n)) \Rightarrow w_{\eta}(n+1)=w_{\eta}(n)+\frac{D}{n}, w_{U}(n+1)=w_{U}(n)-\frac{D}{n}
\end{array}\right\} .
$$

Or its equivalent constrains equation: 


$$
\left\{\begin{array}{l}
\text { if }(\eta(\mathrm{n})>U(n)) \Rightarrow \lambda_{\eta}(n+1)=\lambda_{\eta}(n)-\frac{D}{n} \\
\text { if }(\mathrm{U}(\mathrm{n})>\eta(\mathrm{n})) \Rightarrow \lambda_{\eta}(n+1)=\lambda_{\eta}(n)+\frac{D}{n}
\end{array}\right\},
$$

where $D \in(0,1)$.

As the $D M F$ process advances ( $n$ index increases) the weight's variation $(D / n)$ decreases, resulting in a convergent optimization algorithm of high accuracy.

\section{3.- Optimization of Nonimaging devices.}

A study comparing conventional and dynamic merit functions is carried out to analyze the effectiveness of $D M F$ technique. Thus the difference between conventional optimization and dynamic weights is presented. For this study the following optical systems are selected: concentrating lens, uniformizing lens and a flat LED Luminaire.

The light source employed for the concentrating lens is a 200000 rays collimated random pattern, while the source employed for the uniformizing lens and the flat LED luminaire is a Luxeon Rebel InGaN LED model of 500000 rays.

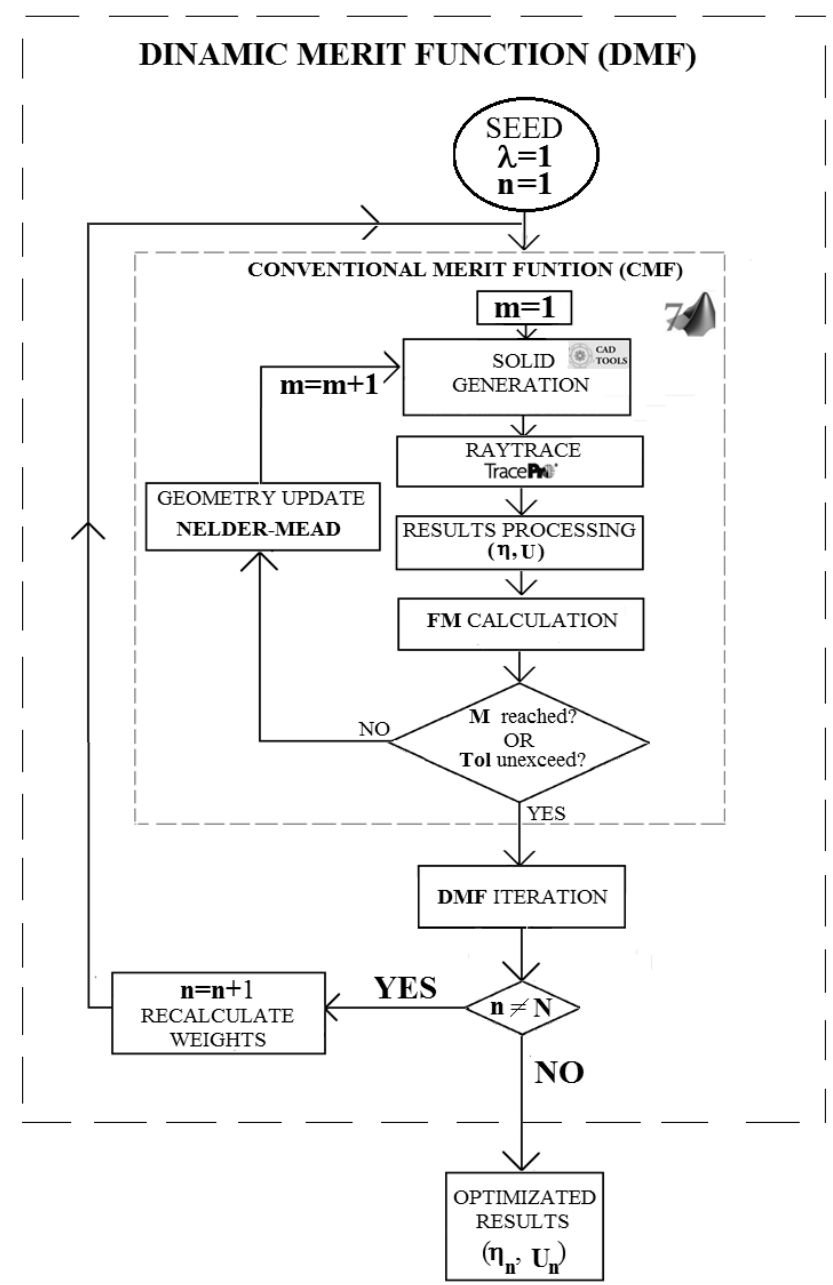

Figure 1. DMF Optimization. Flux diagram. 
The optimization objectives are measured at the detector screen. The work plane subtends $5^{\circ}$ in the concentrating lens case and $60^{\circ}$ angle from the uniformizing lens and flat LED luminaire, the matrix employed to process results is $512 \times 512$ pixels size.

The values reached by the objectives of our merit function should be balanced regardless the difference between the contribution weighted by the merit function [12]. The variation of the weights follows a linear algorithm (5) that ensures the balance between the optimization objectives, the weights increment is set to $D / n$ where $D=0.3$ and $n$ is the $D M F$ iteration index. The $C M F$ tolerance parameters are set to TolFun $=$ Tol $X=10^{-4}$.

The maximum $D M F$ iterations is set to $N=3$, each one equivalent to multiple $C M F$ iterations (from 60 iterations for the uniformizing lens up to 160 for the flat LED luminaire). The execution time required depends heavily on these optimization parameters because the $D M F$ requires $N$ times the $C M F$ execution time. This augment of time is worth as long as the DMF achieves better results with significant improvement of the objectives

\section{3.a- Uniformizing Lens.}

The uniformizing lens is an optical revolving geometry characterized by two radii $\left(R_{1}, R_{2}\right)$, a height distance $H$, a thickness $T$, an aperture angle $\alpha$ and the refractive index $n$ (fig. 2a). The first three variables among them are chosen as parameters the optimization. This optical device is designed to achieve a batwing type emission that ensures a greater rate of uniformity . The initial values are $R_{1}=R_{2}=50 \mathrm{~mm}$ and $H=25 \mathrm{~mm}$.

The dynamic optimization DMF demonstrates its effectiveness by increasing efficiency close to $30 \%$ and uniformity up to $9.5 \%$ compared to the CMF optimization applied to the same uniformizing lens. The final configuration of the uniformizing lens $\left(R_{1}=63.7 \mathrm{~mm} R_{2}=79.9 \mathrm{~mm}\right.$ $H=19.8$ ) , achieved by the $D M F$ optimization (fig. 2), depends not only on the weight's factors but also on the algorithm's initial values. Depending on the initialization seed, the DMF process may take more or less iterations to achieve the best results, while this very factor is specially relevant within the $C M F$ algorithm where it can determine its global effectiveness.
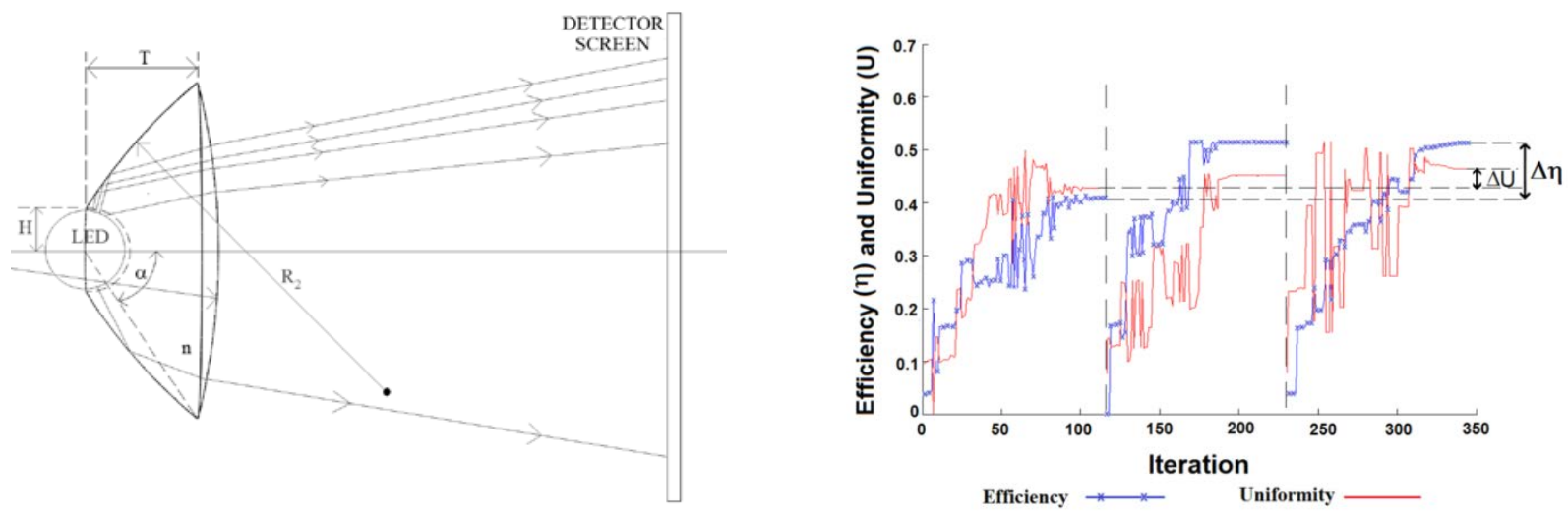

Figure 2. Uniformizing Lens. Optimization parameters: $R_{1}, R_{2}$ and $H$. DMF applied to 3 parameters uniformizing Lens. 


\section{3.b- Flat LED Luminaire .}

The dynamic optimization is now applied to a flat LED technology luminaire. This system is considerably more complex than the previous analyzed. The Luminaire is formed by a LED collimator of depth $D$, joined to a staggered duct that reflects the light to a micro optic distribution matrix (TIR reflector based) described by the entrance radius $R$, the acceptance $\theta_{l}$ and emission angle $\theta_{2}$ (fig.3). These variables $\left(D, R, \theta_{1}, \theta_{2}\right.$ ) conform the parameters of the DMF optimization. The initial values for the luminaire parameters are: $D=15 \mathrm{~mm}, R=2 \mathrm{~mm}, \theta_{1}=\theta_{2}=12^{\circ}$.

The DMF optimization results (fig.7) shows a great improvement ( $\Delta \eta 25 \%, \Delta U 6 \%$ ) compared with the $C M F$ results. This results confirms the suitability of the $D M F$ optimization for complex systems. Optimized parameters establish at followings values: $D=10.4 \mathrm{~mm}, R=1.1 \mathrm{~mm}, \theta_{1}=15.3^{\circ}$, $\theta_{2}=21.4^{\circ}$.The Evolution of the irradiance maps and the corresponding uniformity magnitude of this Flat LED luminaire, as well as the concentrating and uniformizing lenses, can be observed in figure 3.
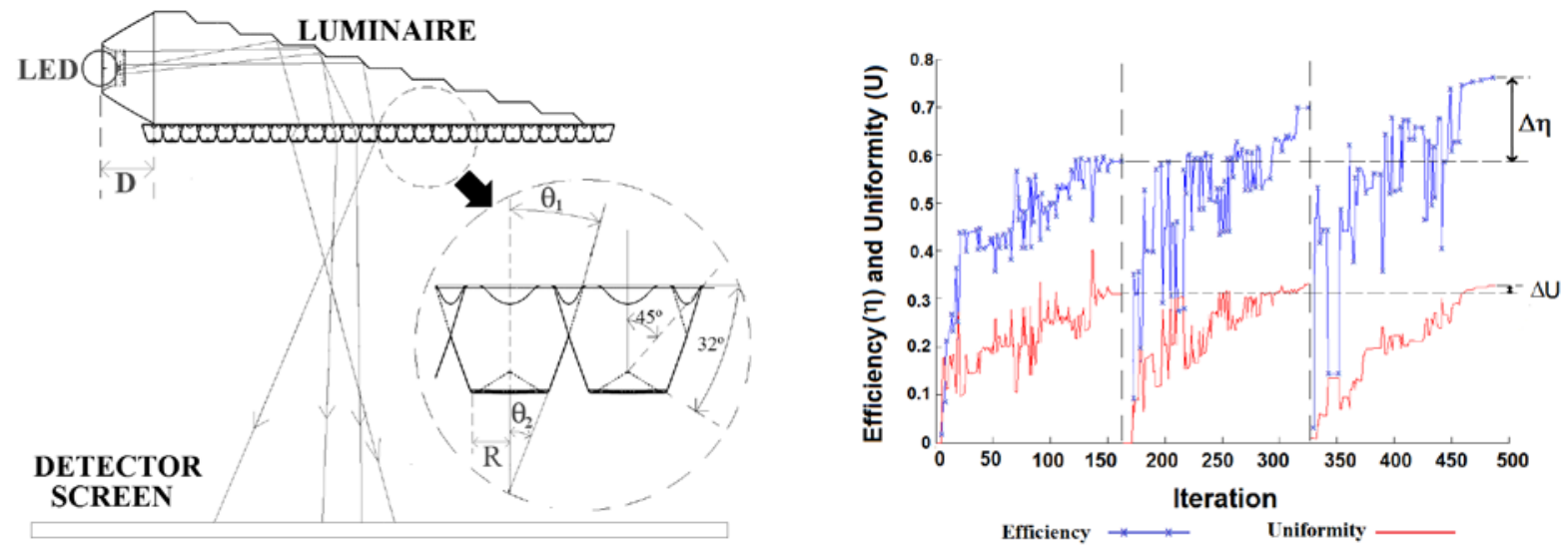

Figure 3. Flat LED Luminaire. Optimization parameters: $D, R, \theta_{1}, \theta_{2}$.

\section{4.- . Dependence on parameters in DMF optimization}

The success of the optimization process depends on several factors, some of them related to the space variable that defines the solid geometry. The number of local minimums in the merit function's space variable is proportional to the optical system's complexity, therefore increasing the number of geometry's parameters enhance the probabilities that optimized solution might be stuck in a local minimum.

To analyze the effect of the number of parameters in the optimization results the DMF will be applied to systems with variable numbers of parameters. A LED collimator device will be optimized taking into account the number of parameters describing its geometry. The collimator design was already employed at the LED Luminaire entrance. The detector will consists on plane that subtends $3^{\circ}$ from the collimator where the uniformity and the efficiency will be evaluated.

In this occasion two different DMF optimizations will be carried out depending on the number of parameters describing the collimator: the first optimization will consider two parameters, depth distance $D$ and the radius $R_{2}$; the second optimization will choose the depth distance $D$ and the radii $R_{2}$ and $R_{3}$ among the geometry parameters. The DMF optimization applied to 2 parameters 
collimator achieves nearly identical results despite of the $M F$ weights' value that changes in every $C M F$ optimization. The results achieved with a $D M F$ process applied on the same collimator but provided with an additional degree of freedom (three parameters) show a notable improvement in terms of efficiency $22.4 \%$ (Table 2) regarding the CMF optimization. DMF optimization, applied to a 3 parameters collimator, improves over the initial static weights optimization, since the first $C M F$ optimization gets stuck in a local minimum of the $M F$ due to the greater complexity of the variables' space compared to the 2 parameters collimator. The 2 parameters optimization choose the collimator depth $D$ and the reflector radius $R$ as variables. The DMF obtains better results compared to $\mathrm{CMF}$ as the system under optimization is described with higher number of parameters. The 4 parameter's DMF optimization achieves an improvement of $\Delta \eta=24.3 \%$ and $\Delta \mathrm{U}=4.1 \%$ compared to 2 parameters Luminaire (Table 1).

Table 1. DMF Results. Influence of number of parameters. LED collimator and flat LED luminaire.

\begin{tabular}{|c|c|c|}
\hline System & $\Delta \eta$ & $\Delta \mathbf{U}$ \\
\hline Collimator (2 vs. 3 parameters) & $22.4 \%$ & $-11.3 \%$ \\
\hline Luminaire (2 vs. 4 parameters) & $24.3 \%$ & $4.1 \%$ \\
\hline
\end{tabular}

\section{5.- Multistart algorithm to flowline technique to nonimaging design}

As we have mention the start point has notorious influence in the optimization process. In the flowline technique sometimes it is needed to compute the Optical Path Lengh $L$ of a ray by means of Fermat principle, it is well known that Fermat principle is a minimum principle and requires a minimum computation in analogous way that optimization process. In that case we have developed a multistart algorithm to compute Optical Path Length $(O P L)$ for refractive and reflective optical systems. It uses the basic Nelder-mead algorithm employed in optimization problems. We have built a vector of starting points with a complete sampling of limits of parameters, in our case the starting point vector completely sample the lens. We force the algorithm to choose the minimum between all starting points sampled in the computation process, included in the vector. Figure 4 shows the relation between $O P L$ computation, $\frac{\text { Multistart } O P L}{\text { Standar } O P L}$ for a simple system with a linear lambertian source a plano convex lens and a square detector located at $1150 \mathrm{~mm}$ to the source. Figure shows difference between MultiStart computations and classical computations which modifies flowline results. 

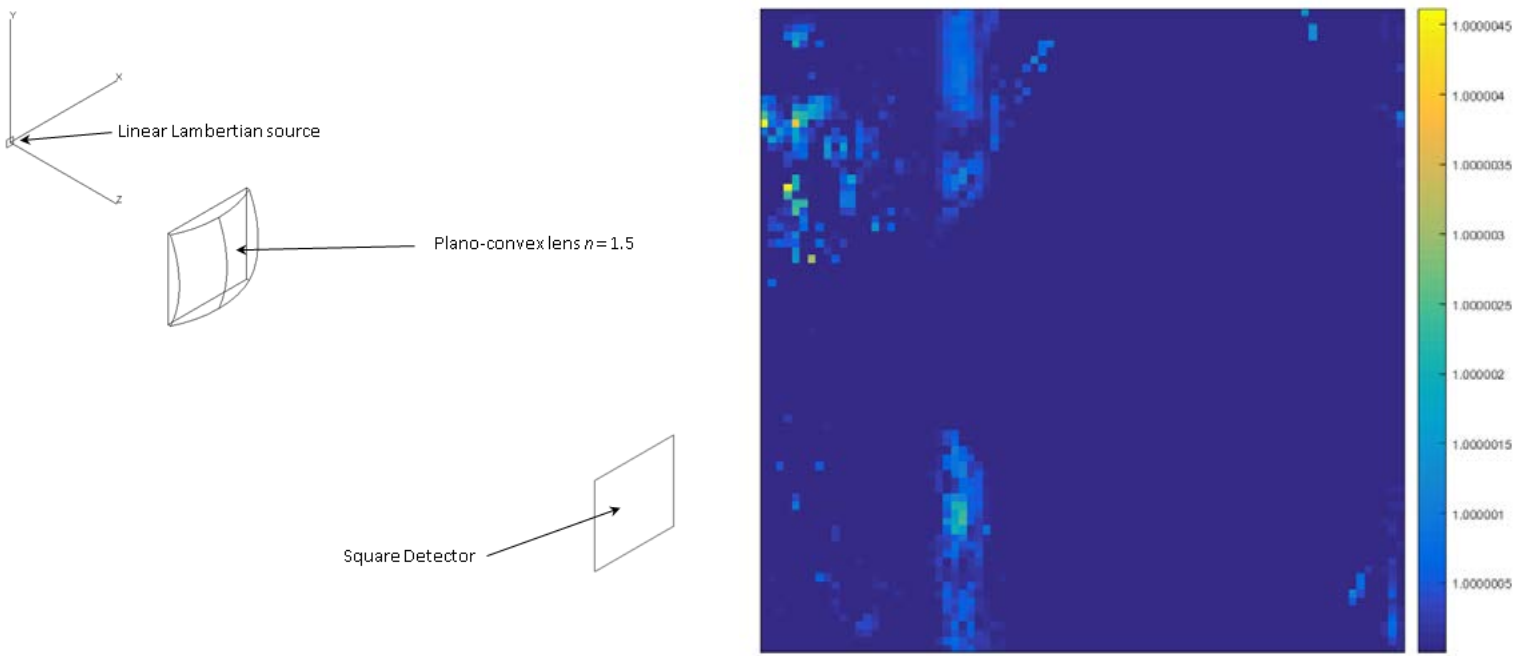

Figure 4. Sketch of the analyzed system to compute OPL, and quotient between MultiStart OPL computation and standard OPL computation of the system

\section{6.- Conclusions}

Optimization technique are well stablished in nonimaging optics design process. Nevertheles Evolutionary or Global techniques are not well developed yet, we have developed and analyzed Dynamic Merit Function and Multistart algorithms applied to nonimaging design technique. Those algorithms shows improvements in the design and corrects the standard algorithm results.

\section{7.- Bibliography}

[1] W. J. Cassarly and M. J. Hayford, "Illumination optimization: the revolution has begun” Proc. SPIE 4832, 258-269 (2002).

[2] Tracepro software, http://www.lambdares.com/.

[3] Lightools software, http://www.opticalres.com/.

[4] W. J. Cassarly, “Illumination merit functions” Proc. SPIE 6670, 142-154 (2007).

[5] J. R. Koshel, “Aspects of Illumination system optimization” Proc. SPIE 5529, 206-217 (2004).

[6] Z. Gao, Y. Wang, G. Gioia and A. M. Cuitiño "A global approach for digital speckle correlation” Annual Conference \& Exposition on Experimental and Applied Mechanics (2002)

[7] J. R. Koshel “Simplex optimization method for illumination design”, Optics Letters, 30 (6), 649651,2005

[8] Y. Jin, T. Okabe and B. Sendhoff "Adapting Weighted Aggregation for Multiobjective Evolution Strategies" Procedings of the Evolutionary Multi-criterion Optimization Conference, EMO 2001, 96 -110, Zurich 2001. 
[9] X. Luo, H. Liu, Z. Lu and Y. Wang, "Automated optimization of an aspheric light-emitting diode lens for uniform illumination", Applied Optics, 50 (20), 3412-3418, 2011

[10] R. J. Koshel, "Fractional Optimization of illumination optics", procedings of SPIE, vol 7061, Novel optical systems design and optimization XI, 2008.

[11] J. C. Lagarias, J. A. Reeds, M. H. Wright, and P. E. Wright "Convergence Properties of the Nelder-Mead Simplex Method in Low Dimensions" SIAM J. Optim., 9(1), 112-147 (1998)

[12] N. E. Shatz and J. C. Bortz, "Optimal design of a nonimaging TIR doublet lens for an illumination system using an LED source” SPIE Proc. of Nonimaging Optics and Efficient Illumination Systems I 5529, 8 (Society of Photo-Optical Instrumentation Engineers, Bellingham, WA, 2004). 